\title{
Psychophysical methodology IV: Phi gamma hypothesis and the method of limits
}

\section{ROBERT M. HERRICK ${ }^{1}$ NA VAL AIR DEVELOPMENT CENTER}

Assuming the phi-gamma hypothesis, deductions concerning the method of limits (ML) are derived. For a given step size, the selection of the initial stimulus for ascending series or for descending series has little effect on the summary statistical measures of the ML. Estimates of $M L$ statistics are derived for different step sizes, and these estimates are used to estimate the mean and standard deviation of the phi-gamma hypothesis. Also considered are how summary statistical measures of the $M L$ are influenced by extremely large and small step sizes and by the definition of a ML threshold.

Assuming the phi-gamma hypothesis, Herrick (1970) derived equations relating means and standard deviations of distributions of the method of limits (ML) and the method of constant stimuli (MCS). In the derivations, the stimulus values for a ML experiment were selected with respect to the axis of symmetry of the cumulative normal curve (phi-gamma hypothesis), although this axis is ordinarily not known. The present paper shows how ML and MCS statistics may be related without assuming that stimuli are spaced with respect to the axis of symmetry.

This paper also considers two other problems in relation to the phi-gamma hypothesis: (1) How do different definitions of a ML threshold influence means and standard deviations of $\mathrm{ML}$ threshold distributions? (2) What are the effects of very large and very small step sizes in the ML?

\section{AXIS OF SYMMETRY AND METHOD}

\section{OF LIMITS STIMULUS VALUES}

A cumulative normal distribution is specified by its mean and standard deviation, $M_{M C S}$ and $\sigma_{M C S}$. The axis of symmetry of the distribution is $\mathrm{M}_{\mathrm{MCS}}$. The spacing (step size) between successive stimuli in a ML experiment equals c. Now, a set of ML stimuli selected with respect to $M_{M C S}$ must include a stimulus equal to $\mathbf{M}_{\mathrm{MCS}}$ or to $\left(\mathrm{M}_{\mathrm{MCS}}-\mathrm{c} / 2\right)$. Otherwise, the ML stimuli do not fall with respect to M MCs.

Fig. 2. Influence of the initial stimulus in the ascending method of limits (AML) on the standard deviation of the AML threshold distribution, $\sigma_{\mathbf{A}}$.

Perception \& Psychophysics, 1970, Vol. 8 (1)

\section{RELATING THE ML AND THE MCS}

Consider a cumulative normal distribution with $M_{M C S}=55.00$ and $\sigma_{\mathrm{MCS}}=10.00$. For this distribution, with INITIAL STIMULUS (SO) IN ASCENDING SERIES IN TERMS OF MMCS ANO $\sigma_{\text {MCS }}$

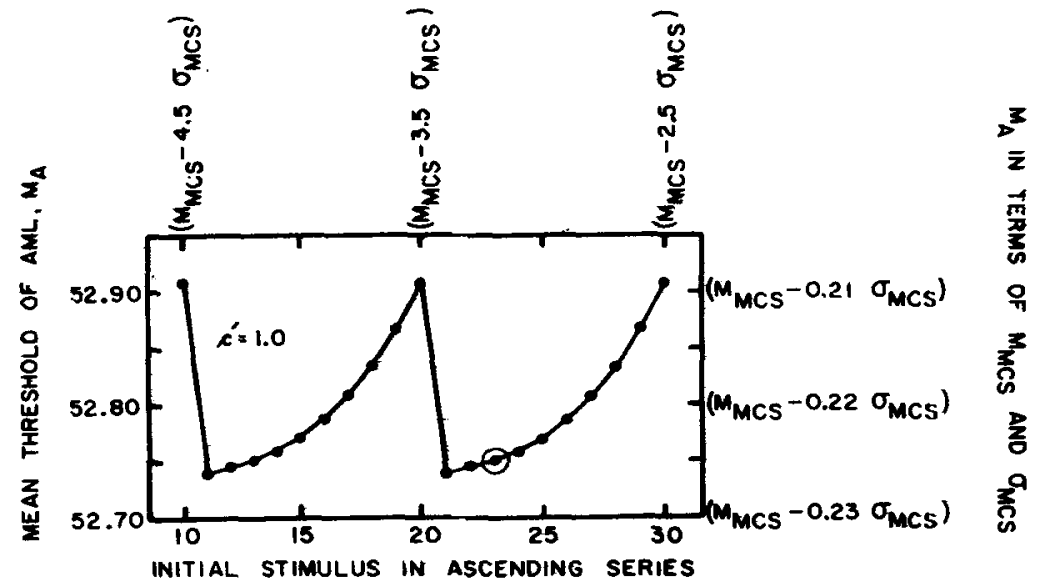

Fig. 1. Influence of the initial stimulus in the ascending method of limits (AML) on the mean of the AML threshold distribution, $M_{A}$. The scales on the bottom and on the left refer to numerical examples. The other two scales give the same information in terms of $M_{\text {MCS }}$ and $\sigma_{\text {MCS. }}$. Circled point refers to the first example discussed in text.

INITIAL STIMULUS (SO) IN ASCENDING SERIES IN TERMS OF MMCS AND $\sigma_{\text {MCS }}$

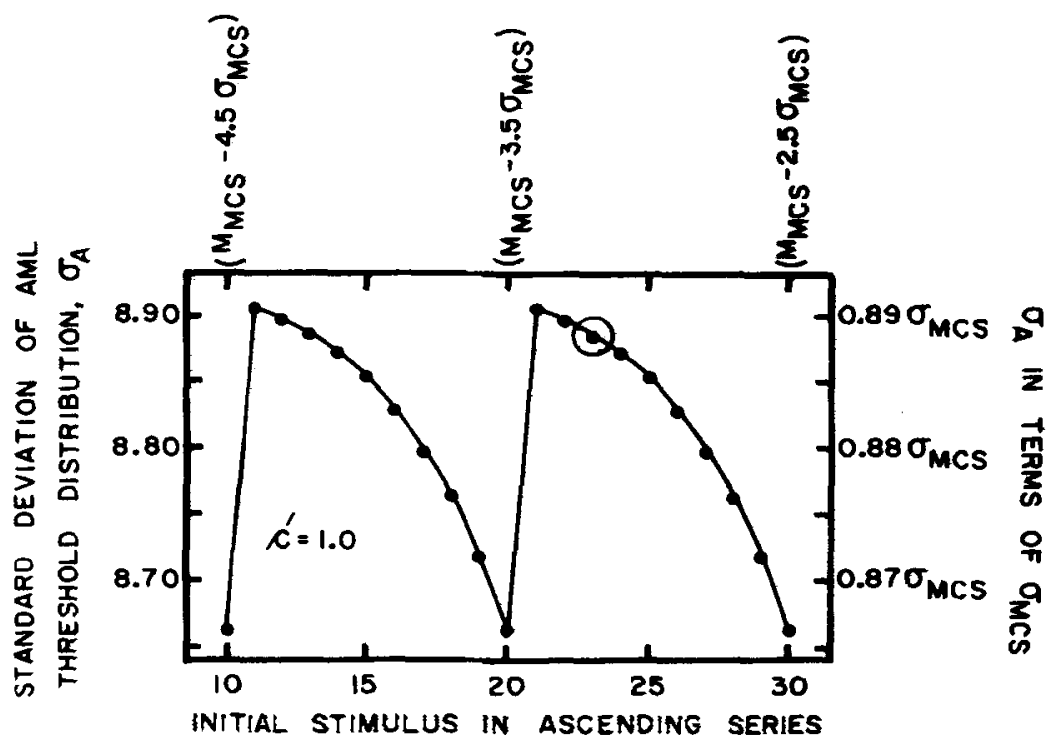

Copyright 1970, Psychonomic Journals, Inc., Austin, Texas 
$1969,1970)$. For example, with an initial stimulus $\left(\mathrm{S}_{0}\right)$ equal to 23 , the set of $\mathrm{ML}$ stimuli is $23,33,43,53,63,73$, and 83 , and $M_{A}=52.752$. (Since none of this set of ML stimuli is equal to 55 or $(55-10 / 2)$, the stimuli are not spaced with respect to $\mathbf{M}_{\mathbf{M}}$ Cs.)

Figure 1 gives the results of the computations. 3 Figure 1 also includes, in the upper and the right-hand scales, a designation of $S_{0}$ and $M_{A}$ in terms of MMCS and $\sigma_{M C S}$. Thus, $S_{0}=23$ is designated as $(55-32)$ or $\left(\mathrm{M}_{\mathrm{MCS}}-3.2 \sigma_{\mathrm{MCs}}\right.$ ), and $\mathrm{M}_{\mathrm{A}}=52.752$ is designated as $(55-2.248)$ or (MCS $\left.-.2248 \sigma_{\mathrm{MCS}}\right)$.

A ML term may also be specified in units of $\sigma_{\mathrm{MCS}}$ by dividing the term by $\sigma_{\text {MCs. }}$. When so specified, the term will carry a prime. Thus, $c / \sigma_{\mathrm{MCs}}=\mathrm{c}^{\prime}$; $\left(\mathrm{M}_{\mathrm{MCS}}-.2248 \sigma_{\mathrm{MCS}}\right) / \sigma_{\mathrm{MCS}}=$ $\mathrm{M}_{\mathrm{MCS}}^{\prime}-.2248$.

Following the process just described for $M_{A}$, the standard deviation for the $A M L$, $\sigma_{A}$, for $c^{\prime}=1.0$, was computed for different $S_{0}$ values. Figure 2 summarizes the calculations.

Figures 1 and 2 indicate that for $c^{\prime}=-1.0$, with $S_{0}=\left(M_{M C S}-2.5 \sigma_{M C S}\right)$ or 1 ess, $M_{A}$ falls between $\left(\mathrm{M}_{\mathrm{MCS}} \mathrm{CS} .226 \sigma_{\mathrm{MCS}}\right)$ and $\left(\mathrm{M}_{\mathrm{MCS}}-.209 \sigma_{\mathrm{MCS}}\right)$, and $\sigma_{\mathrm{A}}$ falls between $.866 \sigma_{\mathrm{MCS}}$ and $.890 \sigma_{\mathrm{MCS}}$. Without introducing much error, the midpoints of the $M_{A}$ and $\sigma_{A}$ ranges may be taken as estimates of $M_{A}$ and $\sigma_{A}$. Thus, for any initial stimulus value at or below $\left(\mathrm{M}_{\mathrm{MCS}}-2.5 \sigma_{\mathrm{MCS}}\right)$, whether or not the $M L$ stimuli are selected with respect to the axis of symmetry of the phi-gamma hypothesis, for $c^{\prime}=1.0$, a good estimate of $\mathrm{M}_{\mathrm{A}}$ is $\left(\mathrm{M}_{\mathrm{MCS}}-.2175 \sigma_{\mathrm{MCS}}\right)$, and a good estimate of $\sigma_{A}$ is $.878 \sigma_{M C S}$. In units of $\sigma_{\mathrm{MCS}}$, the estimate of $\mathbf{M}_{\mathrm{A}}$ is $\left(M_{M}^{\prime} \dot{C} S-.2175\right)$ and the estimate of $\sigma_{A}^{\prime}$ is .878 .

By the procedure just described, estimates of $M_{A}^{\prime}$ and $\sigma_{A}^{\prime}$ were obtained for several $c^{\prime}$ values from $c^{\prime}=0.50$ to $c^{\prime}=2.00$. These estimates were then used to derive equations relating the variables. For example, a plot of the estimates of $\sigma^{\prime}$ vs $c^{\prime}$ was fitted by a straight line with the equation:

$$
\sigma_{\mathrm{A}}^{\prime}=.637+.233 c^{\prime}
$$

Multiplying each term of Eq. 1 by $\sigma_{\mathrm{MCS}}$, and solving for $\sigma_{\mathrm{MCS}}$ :

$$
\sigma_{\mathrm{MCS}}=1.57 \sigma_{\mathrm{A}}-.366 \mathrm{c} \text {, }
$$

which gives an estimate of $\sigma_{\mathrm{MCS}}$ when $\sigma_{\mathrm{A}}$ and $c$ are known.

Similarly, a plot involving $\mathbf{M}_{\mathbf{A}}^{\prime}$ was fitted by a straight line with the equation:

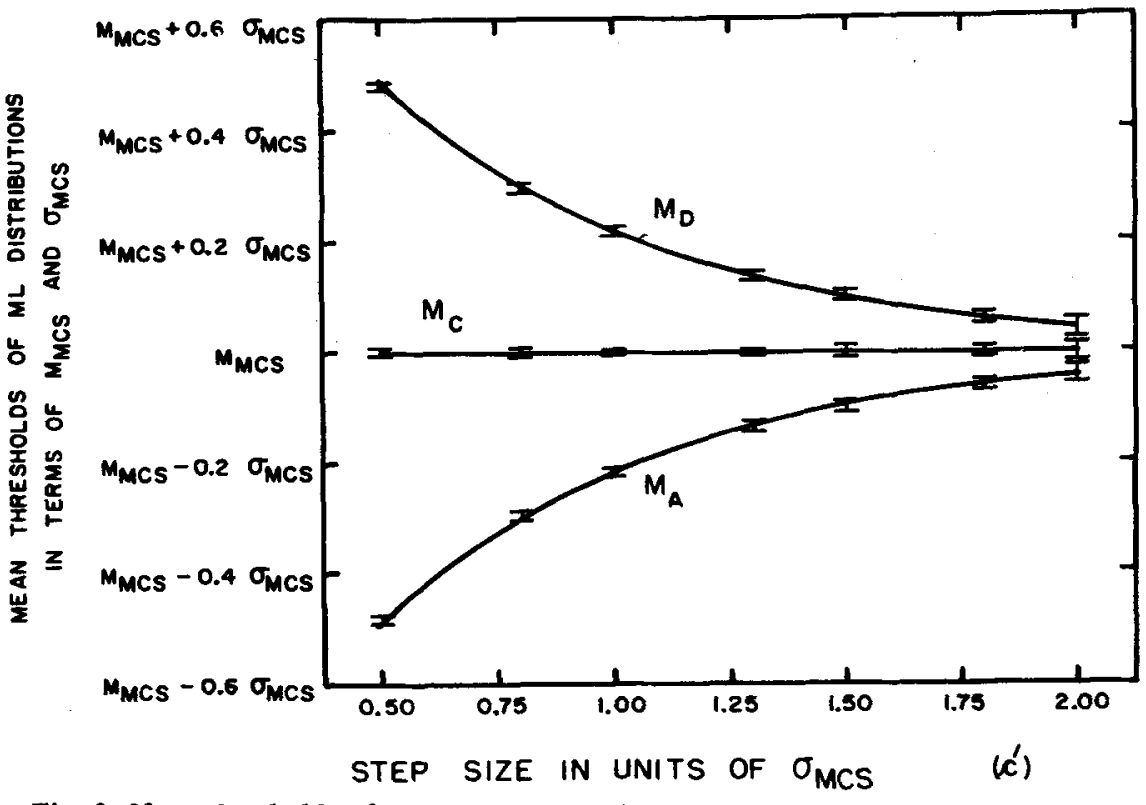

Fig. 3. Mean thresholds of AML, DML, and CML threshold distributions, in terms of $\mathbf{M}_{M C S}{ }^{a n d} \sigma_{M C S}$, as a function of step size. Each pair of short horizontal lines represents the range of mean threshold values expected. The curves represent equations given in the text.
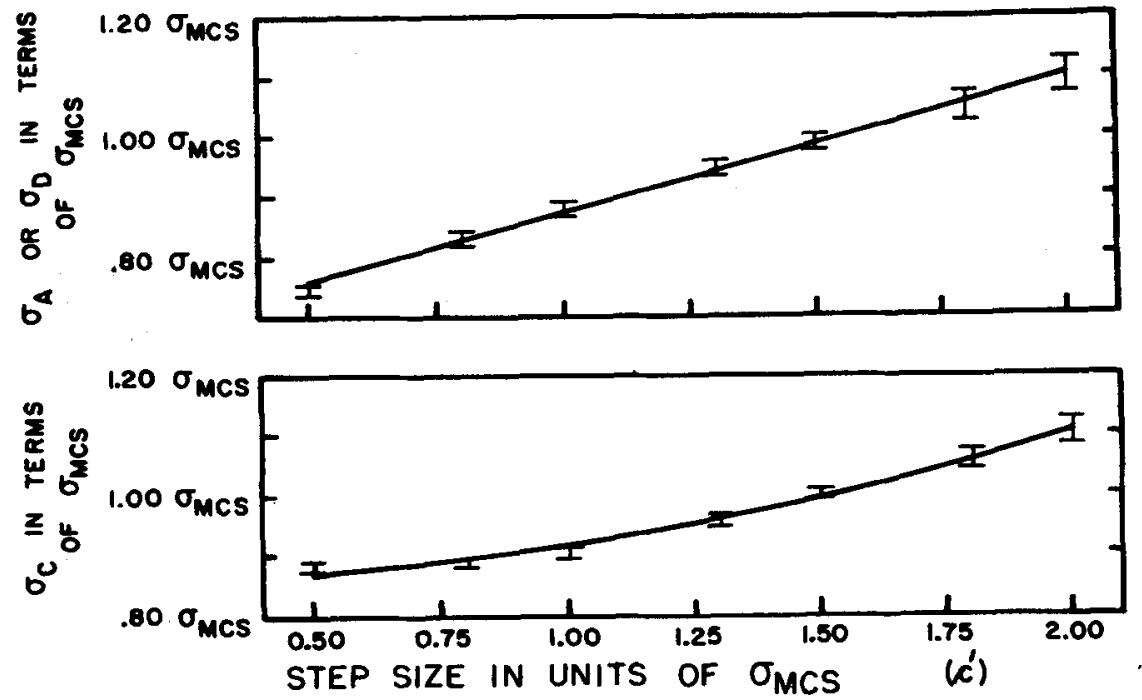

Fig. 4. Standard deviations of AML, DML, and CML threshold distributions in terms of $\sigma_{\mathrm{MCS}}$ as a function of step size. Each pair of short horizontal lines represents the range of standard deviations expected, and the curves represent equations given in the text.

Table 1

Summary Statistics of Method of Limits Threshold Distributions

\begin{tabular}{|c|c|c|c|}
\hline \multirow[b]{2}{*}{ Surnmary Statistic } & \multicolumn{3}{|c|}{ Definition of Threshold } \\
\hline & $\begin{array}{c}\text { Standard } \\
\text { Definition } \\
\end{array}$ & Definition $L$ & Definition NL \\
\hline $\begin{array}{l}\text { Mean Threshold of AML } \\
\text { Mean Threshold of DML } \\
\text { Mean Threshold of CML** } \\
\text { Standard Deviation of AML } \\
\text { Standard Deviation of DML } \\
\text { Variance of CML** }\end{array}$ & $\begin{array}{l}M_{A} \\
M_{D} \\
M_{C} \\
\sigma_{A} \\
\sigma_{D} \\
\sigma_{C}^{2}\end{array}$ & $\begin{array}{l}\mathrm{M}_{\mathrm{A}}+\mathrm{c} / 2 \\
\mathrm{M}_{\mathrm{D}}-\mathrm{c} / 2 \\
\mathrm{M}_{\mathrm{C}} \\
\sigma_{\mathrm{A}} \\
\sigma_{\mathrm{D}} \\
\sigma_{C}^{2}+c^{2 / 4} \\
-\mathrm{c}\left[\left(\mathrm{M}_{\mathrm{D}}-\mathrm{M}_{\mathrm{A}}\right) / 2\right]\end{array}$ & $\begin{array}{l}\mathrm{M}_{\mathrm{A}}-\mathrm{c} / 2 \\
\mathrm{M}_{\mathrm{D}}+\mathrm{c} / 2 \\
\mathrm{M}_{\mathrm{C}} \\
\sigma_{\mathrm{A}} \\
\sigma_{\mathrm{D}} \\
\sigma_{\mathrm{C}}^{2}+\mathrm{c}^{2} 2 / 4 \\
+\mathrm{c}\left[\left(\mathrm{M}_{\mathrm{D}}-\mathrm{M}_{\mathrm{A}}\right) / 2\right]\end{array}$ \\
\hline
\end{tabular}
in Relation to the Definition of a Threshold*

* See text for definitions

** With an equal number of $A M L$ and DML thresholds 

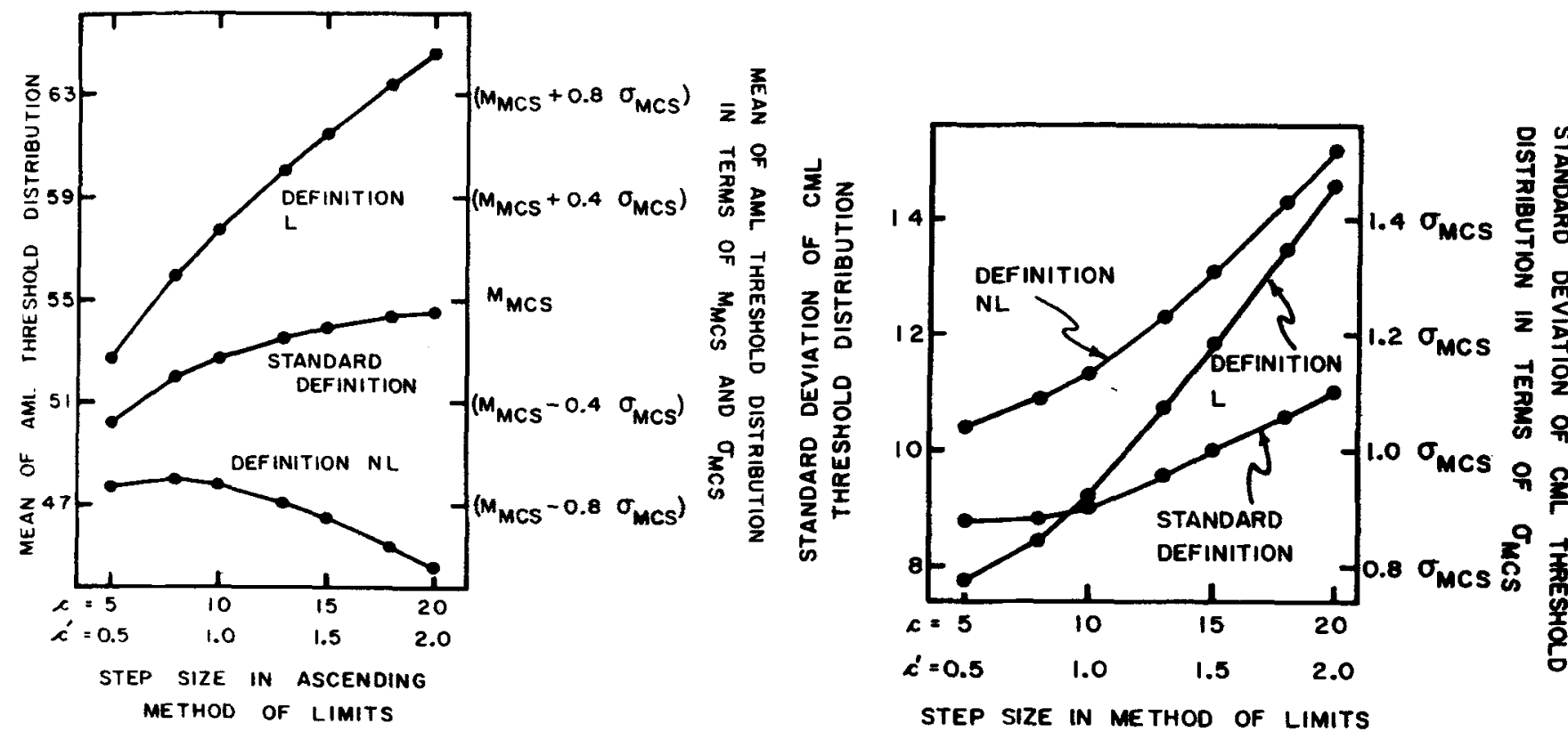

Fig. 5. Influence of the definition of a threshold on the mean threshold of the AML. The left scale gives the mean threshold for the numerical example. The right scale gives the same measure in terms of $\mathrm{MMCS}_{\mathrm{MC}}$ and $\sigma_{\mathrm{MCS}}$. See text for definitions.

$$
\log \left(M_{M C S}^{\prime}-M_{A}^{\prime}\right)=.041-.703 c^{\prime},(3) \quad \sigma_{M C S}=.587\left[\sigma_{C}+\left(\sigma^{2} C-.215 c^{2}\right)^{1 / 2}\right] .
$$

which can be written as

$$
\begin{array}{ll}
\log \left[\mathbf{M}_{M C S}-\mathbf{M}_{\mathrm{A}}\right] & \log \left[\mathbf{M}_{\mathbf{D}}-\mathbf{M}_{\mathbf{M C S}}\right] \\
=\left[\log \sigma_{M C S}+.041\right]-\left[.703 / \sigma_{M C S}\right] \mathrm{c} . & =\left[\log \sigma_{M C S}+.041\right]-\left[.703 / \sigma_{M C S}\right] \mathrm{c}
\end{array}
$$

By substituting in Eq. 4 the value of $\sigma_{\mathrm{MCS}}$ obtained by Eq. 2, an estimate of MMCS may be obtained when $M_{A}, c$, and $\sigma_{A}$ are known.

The procedure described above was also followed for the descending method of limits (DML) and for the combined method of limits (CML). The equations derived are

$$
\sigma_{\mathrm{MCS}}=1.57 \sigma_{\mathrm{D}}-.366 \mathrm{c} .
$$

$$
\mathbf{M}_{\mathrm{MCS}}=\mathbf{M}_{\mathrm{C}} \text {. }
$$

In Eqs. 5-8 the subscripts $D$ and $C$ refer to the DML and the CML threshold distributions, respectively.

Figures 3 and 4 present curves representing the equations given above, along with the range of values expected. For example, at $c^{\prime}=1.0, M_{A}$ ranges from ( $\left.\mathrm{M} \mathrm{M} \mathrm{C} \mathrm{S}_{\mathrm{MCS}} .226 \sigma_{\mathrm{MCS}}\right) \mathrm{to}$ (MMCS $-.209 \sigma_{\mathrm{MCS}}$ ), as indicated in

Table 2

Summary Statistics of Method of Limits Threshold Distributions for a Very Large Step Size*

\begin{tabular}{llll}
\hline & \multicolumn{3}{c}{ Definition of Threshold** } \\
\cline { 2 - 4 } Summary Statistic & $\begin{array}{c}\text { Standard } \\
\text { Definition }\end{array}$ & $\begin{array}{c}\text { Definition } \\
\mathrm{L}\end{array}$ & $\begin{array}{c}\text { Definition } \\
\text { NL }\end{array}$ \\
\hline Mean Threshold of AML & $\mathrm{S}_{\mathrm{S}}+\mathrm{c} / 2$ & $\mathrm{~S}_{\mathrm{g}}$ & $\mathrm{S}_{\mathrm{S}}$ \\
Mean Threshold of DML & $\mathrm{S}_{\mathrm{S}}+\mathrm{c} / 2$ & $\mathrm{~S}_{\mathrm{S}}$ & $\mathrm{S}_{\mathrm{g}}$ \\
Mean Threshold of CML*** & $\mathrm{S}_{\mathrm{S}}+\mathrm{c} / 2$ & $\mathrm{~S}_{\mathrm{S}}+\mathrm{c} / 2$ & $\mathrm{~S}_{\mathrm{S}}+\mathrm{c} / 2$ \\
Standard Deviation of AML & 0 & 0 & 0 \\
Standard Deviation of DML & 0 & 0 & 0 \\
Standard Deviation of $\mathrm{CML}$ & 0 & $\mathrm{c} / 2$ & $\mathrm{c} / 2$ \\
\hline
\end{tabular}

* $S_{S}$ is equal to or less than a stimulus with an associated $p$ value of $.00 ; S_{g}$ is equal to or greater than a stimulus with an associated $p$ value of 1.00 .

** See text for definitions.

*** With an equal number of $A M L$ and DML thresholds.
Fig. 3 by the pair of short horizontal lines. Figures 3 and 4 illustrate how ML measures vary as a function of step size and show the fit of Eqs. 1-8 to the calculated data.

\section{INFLUENCE OF DEFINITION OF ML THRESHOLD}

In the above analyses and in earlier papers (Herrick, 1967, 1969, 1970), a ML threshold is defined as the stimulus value midway between the stimulus at which the ML series terminated and the preceding stimulus in the series (standard definition). It is of interest to consider the influence of two other definitions of the ML threshold on the summary statistical measures of ML distributions. Definition $\mathrm{L}$ defines a $\mathrm{ML}$ threshold as the stimulus at which the last judgment in each ML series occurred. Definition NL defines a ML threshold as the stimulus at which the next-to-last judgment in each ML series occurred.

Summary statistical measures for the three definitions are given in Table 1. Figures 5 and 6 illustrate the influence of the definitions on the mean threshold of the AML and on the standard deviation of the CML, assuming the phi-gamma hypothesis.

\section{VERY LARGE AND VERY SMALL STEPS IN ML}

When the step size is very large, two stimuli cover the range from $p=.00$ (the probability of a "Yes" response is zero) to $\mathrm{p}=1.00$ (the probability of a "Yes" is 1.00). Table 2 gives the summary statistical 
measures that result from a very large step size.

When the step size is very small, comparable statistical measures are the same for all three definitions of a threshold. If $S_{a}$ is a stimulus with an associated $p$ value just greater than zero, and $S_{z}$ is a stimulus with an associated $p$ value just less than unity: the mean threshold of the AML is $S_{a}$, the mean threshold of the DML is $S_{\mathrm{Z}}$, the mean threshold of the CML is $\left(S_{a}+S_{z}\right) / 2$, the standard deviation of the AML and of the DML distribution is approximately zero, and the standard deviation of the CML distribution is $\left(S_{2}-S_{2}\right) / 2$.

\section{REFERENCES}

HERRICK, R. M. Psychophysical methodology: Comparison of threshold of the method of limits and of the method of constant stimuli. Perceptual \& Motor Skills, 1967, 24, 915-922. HERRICK, R. M. Psychophysical me thodology: Comparisons within the method of limits. Perceptual \& Motor Skills, 1969, 28, 503-514.

HERRICK, R. M. Psychophysical methodology: Deductions from the phi-gamma hypothesis and related hypotheses. Perception \& Psychophysics, 1970, 7, 73-78.

\section{NOTES}

1. Address: Aerospace Medical Research Department, Naval Air Development Center, Johnsville, Warminster, Pennsylvania 18974.
2. In this example, $p$ values, i.e., the probability of "Yes" responses, at 30 and below are taken as .0000 and $p$ values at 80 and above are taken as 1.0000 . For a cumulative normal curve with $\mathrm{MMCS}_{\mathrm{MCS}}=55.00$ and $\sigma_{\mathrm{MCS}}=10.00$ the value of 30 is 2.5 standard deviations below the mean so the true $p$ value is .0062097 . [In a previous analysis (Herrick, 1970), p was considered to be .0000 at 4.0 standard deviations below the mean.]

3. $S_{0}$ values from 10 to 30 were included to illustrate the cyclical changes in $M_{A}$ as a function of $\mathrm{S}_{0}$. The cyclical effect occurs because of the identity of stimuli with $p$ values greater than zero in sets of ML stimuli with $S_{0}$ values differing by $1 c, 2 c$, or any multiple of c. Thus, a set of stimuli $30,40,50,60,70,80$ gives the same $M_{A}$ as a set $20,30,40,50,60,70,80$, when $p=.00$ at 30 and below. 